\title{
AMD3100 improves ovariectomy-induced osteoporosis in mice by facilitating mobilization of hematopoietic stem/progenitor cells
}

\author{
Jin Young Im Im,2,3, Woo-Kie Min $^{4, \#}$, Min Hee Park ${ }^{1,2,3}$, NamOh Kim ${ }^{1,2,3}$, Jong Kil Lee ${ }^{1,2,3}$, Hee Kyung Jin ${ }^{1,5}$, Je-Yong Choi ${ }^{3,6}$, \\ Shin-Yoon Kim ${ }^{4}$ \& Jae-sung Bae, \\ ${ }^{1}$ Stem Cell Neuroplasticity Research Group, Kyungpook National University, ${ }^{2}$ Department of Physiology, Cell and Matrix Research \\ Institute, School of Medicine, Kyungpook National University, ${ }^{3}$ Department of Biomedical Science, BK21 Plus KNU Biomedical \\ Convergence Program, Kyungpook National University, Daegu 700-842, ${ }^{4}$ Department of Orthopaedic Surgery, Kyungpook National \\ University Hospital, Daegu 700-721, ${ }^{5}$ Department of Laboratory Animal Medicine, College of Veterinary Medicine, Kyungpook National \\ University, Daegu 700-721, ${ }^{6}$ Department of Biochemistry and Cell Biology, School of Medicine, Kyungpook National University, Daegu \\ 702-701, Korea
}

\begin{abstract}
Inhibition of an increase of osteoclasts has become the most important treatment for osteoporosis. The CXCR4 antagonist, AMD3100, plays an important role in the mobilization of osteoclast precursors within bone marrow (BM). However, the actual therapeutic impact of AMD3100 in osteoporosis has not yet been ascertained. Here we demonstrate the therapeutic effect of AMD3100 in the treatment of ovariectomy-induced osteoporosis in mice. We found that treatment with AMD3100 resulted in direct induction of release of SDF-1 from BM to blood and mobilization of hematopoietic stem/progenitor cells (HSPCs) in an osteoporosis model. AMD3100 prevented bone density loss after ovariectomy by mobilization of HSPCs, suggesting a therapeutic strategy to reduce the number of osteoclasts on bone surfaces. These findings support the hypothesis that treatment with AMD3100 can result in efficient mobilization of HSPCs into blood through direct blockade of the SDF-1/CXCR4 interaction in BM and can be considered as a potential new therapeutic intervention for osteoporosis.

[BMB Reports 2014; 47(8): 439-444]
\end{abstract}

\section{INTRODUCTION}

Bone remodeling is required for balance between bone-forming osteoblasts and bone resorbing osteoclasts $(1,2)$. Destruction of this balance may lead to the development of bone dis-

${ }^{*}$ Corresponding author. Tel: +82-53-420-4815; Fax: +82-53-4243349; E-mail: jsbae@knu.ac.kr

${ }^{\#}$ These authors contributed equally to this work.

http://dx.doi.org/10.5483/BMBRep.2014.47.8.159

Received 10 July 2013, Revised 22 July 2013,

Accepted 19 November 2013

Keywords: AMD3100, Hematopoietic stem/Progenitor cell, Mobilization, Osteoclast, Osteoporosis ease, such as osteoporosis, which is characterized clinically by low bone density and a consequent increase in the risk of fracture, and pathologically by a decrease in bone mineral density (BMD) and an increase in osteoclastic bone resorption $(3,4)$.

Osteoclasts are somatic cells that differentiate from hematopoietic stem/progenitor cells (HSPCs) (5). Previous study has shown that sphingosine-1-phosphate (S1P), a lipid mediator enhanced in blood, regulates the recruitment of osteoclast precursors from bone marrow (BM) into blood. Treatment with the S1P agonist resulted in relief of bone loss through the reduction of osteoclast deposition onto bone surfaces (6). This study led us to speculate that a more effective approach to the recruitment of endogenous HSPCs into blood in patients with osteoporosis could be via an important therapy involving the decline of osteoclasts differentiated from HSPCs in BM.

Chemokine stromal cell-derived factor-1 (SDF-1, also termed CXCL12), the most powerful chemoattractant of both human and murine HSPCs, and its major receptor, CXCR4, are key players in HSPC mobilization $(6,7)$. The number of circulating stem cells can be significantly increased by mobilizing them from the BM to the peripheral blood with cytokines, chemokines, or small molecule inhibitors (8). Due to its potency, granulocyte colony-stimulating factor (G-CSF) is currently the most widely used agent for induction of HSPC mobilization (9). However, mobilization of HSPCs by G-CSF occurs through suppression of osteoblast cells and decreasing expression of SDF-1 on osteoblasts, resulting in major impairment of HSC retention in BM and reduced bone formation $(9,10)$. It is also associated with a clinically significant osteopenia, characterized by an increase in osteoclast activity and a decrease in BMD (11-13). In order to overcome these obstacles, we used AMD3100, which inhibits SDF-1-mediated mobilization through direct blockade of the chemokine binding to its receptor, CXCR4, without impairment of osteoblast function $(10,14,15)$ in a model of postmenopausal osteoporosis. AMD3100 has recently been reported to enhance recruitment of CXCR4-dependent HSPCs by inducing an increase in the level of SDF-1 in blood secreted

ISSN: 1976-670X (electronic edition)

Copyright (C) 2014 by the The Korean Society for Biochemistry and Molecular Biology

(c) This is an open-access article distributed under the terms of the Creative Commons Attribution Non-Commercial License (http://creativecommons.org/licenses/by-nc/3.0) which permits unrestricted non-commercial use, distribution, and reproduction in any medium, provided the original work is properly cited. 
from BM stromal cells $(15,16)$.

Based on these concepts and findings, this study was designed to determine whether AMD3100 has the capacity to mobilize HSPCs, resulting in a decrease in the number of osteoclasts in BM. If so, use of this molecule might have a beneficial effect on ovariectomy (OVX)-induced osteoporosis in mice.

\section{RESULTS}

To determine the effect of AMD3100 on OVX mice, we injected OVX mice with AMD3100. The injection protocol is described in Fig. 1A. Recent studies have demonstrated direct induction of release of SDF-1 into the circulation and an increase in mobilization of HSPCs by treatment with AMD3100 (14). Therefore, we performed an analysis in order to determine whether treatment with AMD3100 could induce release of SDF-1 and whether it is associated with recruitment of HSPCs. Consistent with previous results (14), we found that levels of functional SDF-1 decreased in BM and increased in plasma of the AMD3100-treated group (Fig. 1B and C). These

(A)

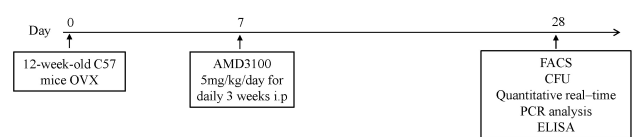

(B)
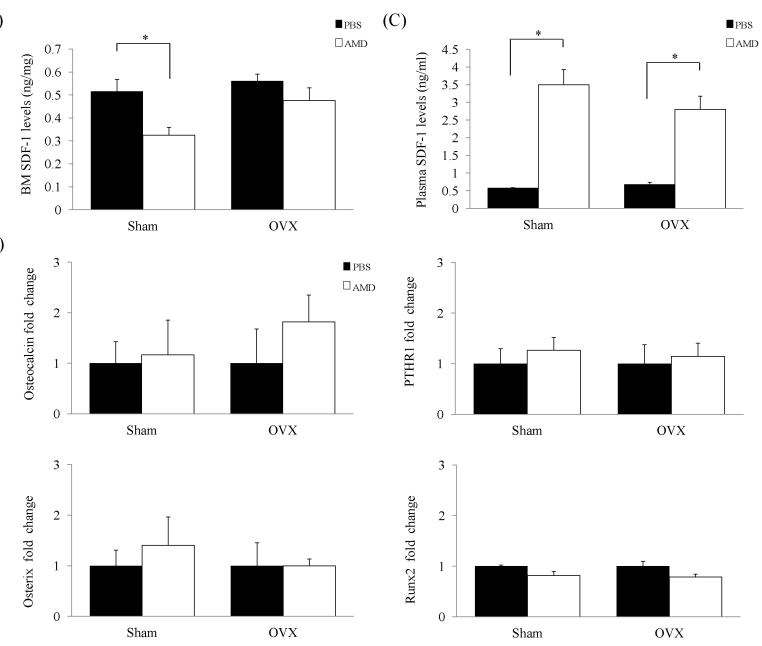

Fig. 1. SDF-1 level increased in blood and did not affect osteoblasts. (A) Timeline of the experimental design of this study. Twelve-week-old C57BL/6 mice $(n=40)$ were divided into four groups (Sham/PBS group [n = 10]; Sham/AMD3100 group $[\mathrm{n}=$ 10]; OVX/PBS group [ $\mathrm{n}=10]$; OVX/AMD3100 group $[\mathrm{n}=10]$ ). (B, C) Steady-state homeostasis fold change in levels of SDF-1 was evaluated in mouse plasma and BM supernatants after administration of PBS or AMD3100. AMD3100 induced release of functional SDF-1 to plasma. (D) Expression of Osteocalcin, PTHR1, Osterix, and Runx2 was analyzed by Real-Time PCR from BM of OVX and sham mice. Data represent mean \pm SEM (Student's t-test. $\mathrm{n}=4-5$ per group). ${ }^{*} \mathrm{P}<0.05$ compared with AMD3100 treated mice or matched control. results indicate that treatment with AMD3100 resulted in an increase in SDF-1 in blood and might affect HSPCs mobilization from BM to blood. Next, we investigated osteoblast lineage-specific genes, including Osteocalcin, PTHR1, Osterix, and Runx2 in order to determine whether AMD3100 has an effect on osteoblast. However, no significant differences in the levels of osteoblast lineage-specific genes were observed between the groups (Fig. 1D). These results confirmed that AMD3100 induced an increase in levels of SDF-1 in blood and did not alter osteoblasts of OVX mice.

To determine whether AMD3100 can mobilize HSPCs from $\mathrm{BM}$ in OVX mice, we administered AMD3100 or phosphate-buffered saline (PBS) to OVX for 21 days. As shown in Fig. 2A, the number of hematopoietic colony-forming unit (CFU) cells showed a significant increase in AMD3100-treated OVX mice compared to PBS-treated OVX mice. Flow cytometric analysis was performed for evaluation of the percentage of HSPCs in BM. As expected, the percentage of Lin ${ }^{-} \mathrm{Sca}-1^{+}$ ${\mathrm{C}-\mathrm{Kit}^{+}}^{+}$(LSK) cells showed a decrease in AMD3100-treated OVX mice, compared with PBS-treated OVX mice in BM, although this did not reach statistical significance (Fig. 2B). These data indicate that AMD3100 induces mobilization of HSPCs from BM to blood in a model of OVX-induced osteoporosis.

To investigate the effect of AMD3100 on different bone pa-

(A)

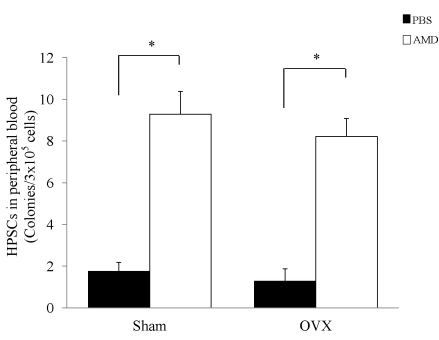

(B)
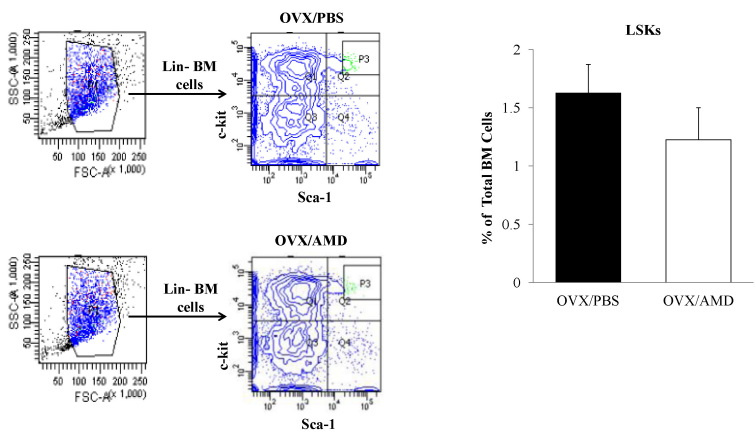

Fig. 2. AMD3100 mobilized HSPCs to blood in an OVX model. (A) We analyzed the effects of AMD3100 on HSPCs mobilization by CFU assay in blood. (B) Flow cytometry analysis of Lin'Sca- $1^{+}$c-kit ${ }^{+}$ cells in BM of PBS-treated OVX and AMD3100-treated OVX mice. Data represent mean \pm SEM (Student's t-test. $n=4-5$ per group). $* P$ $<0.05$ compared with AMD3100 treated mice or matched control. 
rameters, we performed micro-CT analysis for the assessment of $\mathrm{BMD}$, bone mineral content $(\mathrm{BMC})$, bone volume fraction (BVF), tissue mineral density (TMD), trabecular number (Tb.N.), trabecular separation (Tb.Sp.), cortical bone mineral density (Cr.BMD) and cortical bone mineral content (Cr.BMC). Increased bone density was observed in AMD3100-treated OVX mice compared with PBS-treated OVX mice (Fig. 3A). BMD showed an increase in AMD3100-treated OVX mice compared with PBS-treated OVX mice; however, BVF, BMC, TMD, Tb.N., Tb.Sp., Cr.BMD and Cr.BMC were similar between the groups (Fig. 3B).

Osteoporosis is likely the result of osteoclastic deposition rather than osteoblastic defects $(3,4)$. Therefore, in order to determine whether mobilization of AMD3100 affected osteoclast differentiation, we first examined HSPCs differentiation into mature functional osteoclasts by treatment with AMD3100 (17). According to the result, AMD3100 did not affect osteoclast differentiation (Fig. 3C). We also performed tartrate resistant acid phosphatase (TRAP) staining for detection of osteoclasts in the trabecular region of OVX mice. The number and size of TRAP $^{+}$active osteoclasts (black arrowhead) showed a decrease in the trabecular region in AMD3100-treated OVX mice (Fig. 3D). Histomorphometric analysis was performed for determination of the number of osteoclasts. A de- crease in the number of osteoclasts was observed in AMD3100-treated OVX mice compared with PBS-treated OVX mice (Fig. 3E). Next, we performed H\&E staining in order to determine the effect of AMD3100 on the number of osteoblasts. Our findings showed that osteoblast numbers did not change significantly among the AMD treatment groups (Fig. 3F). Taken together, the data presented here demonstrate that treatment with AMD3100 induced mobilization of BM-derived HSPCs into blood, leading to amelioration of bone loss in a model of OVX-induced osteoporosis by reducing the number of osteoclasts attached to the bone surface.

\section{DISCUSSION}

Previous studies have demonstrated a unique role of AMD3100 in the rapid mobilization of osteoclast precursors, HSPCs from BM to blood $(9,10)$. However, the actual therapeutic impact of AMD3100 on pathology of osteoporosis and its mechanism of action have not been determined. AMD3100, a small bicyclam derivative, selectively inhibits binding of SDF-1 in osteoblasts to its receptor, CXCR4, in HSPCs, resulting in rapid mobilization of HSPCs (14). AMD3100-induced levels of SDF-1 can also induce mobilization of HSPCs (18). The data presented here suggest that
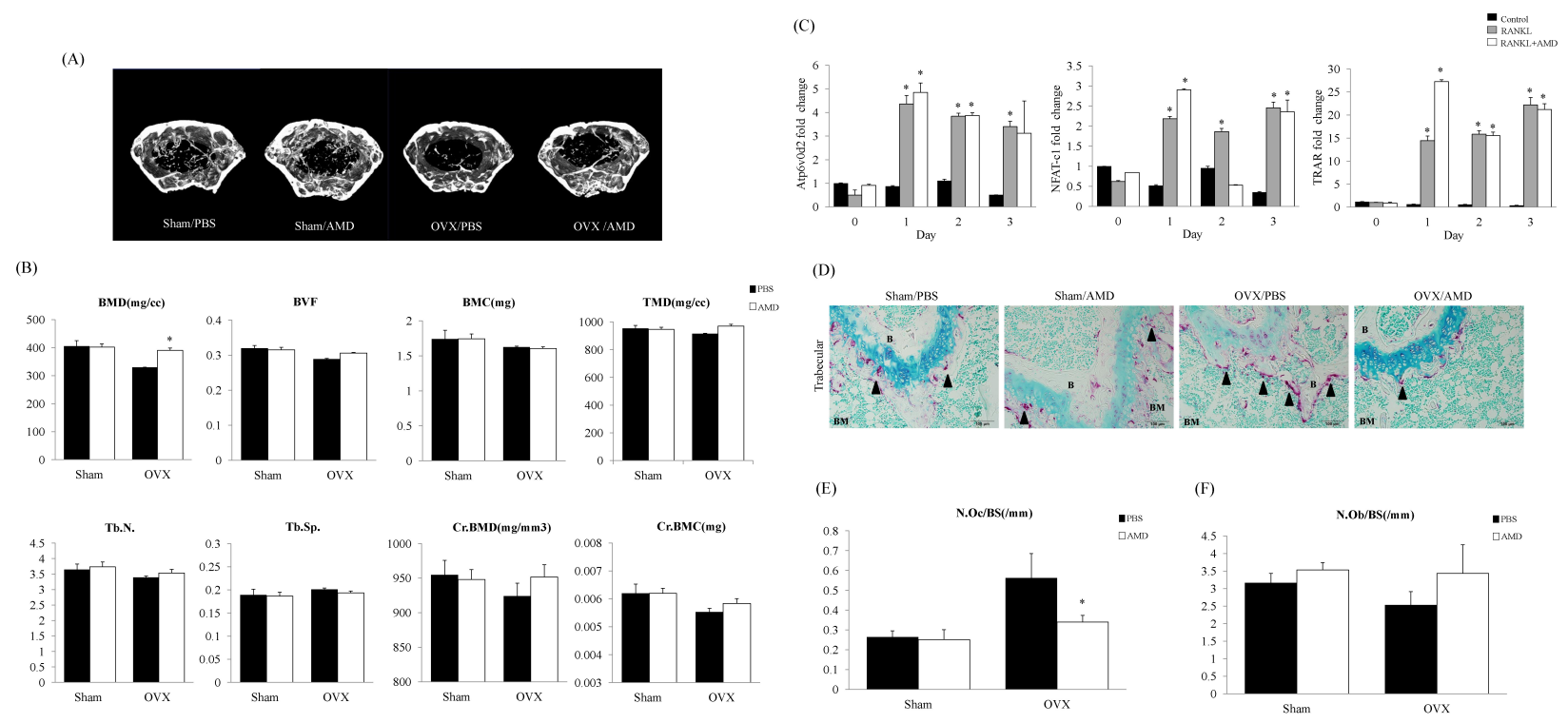

Fig. 3. AMD3100 relieves OVX-induced bone loss through reduction of osteoclast number onto the bone surface. (A) Representative micro-CT images of the distal femurs in each group are shown. (B) Each graph represents BMD, BVF, BMC, TMD, Tb.N., Tb.Sp., Cr.BMD and Cr.BMC in total analysis of cortical and trabecular bone. (C) The effects of AMD3100 on the expression of genes associated with osteoclast differentiation by quantitative real-time PCR. Total RNA was extracted from BM cultures treated with or without RANKL in the presence or absence of $25 \mathrm{\mu g} / \mathrm{ml}$ of AMD3100 for 3 days. Data represent mean \pm SEM (Anova, Tukey's HSD test. $\mathrm{n}=3$ per group). *P < 0.05 compared with AMD3100-treated media or matched control. (D) Representative pictures showing osteoclasts in the trabecular region of OVX mice. Reduction of multi-nucleated TRAP ${ }^{+}$osteoclasts was detected in BM. Arrowheads indicate active TRAP ${ }^{+}$osteoclasts stained in red (original magnification, $\times 20$, Scale bar $100 \mu \mathrm{m})$. (E) Histogram representing the osteoclast number/bone surface [N.Oc/BS (/mm)] and (F) the osteoblast number/bone surface [N.Ob/BS $(/ \mathrm{mm})]$. Data indicate mean \pm SEM (Student's t-test. $\mathrm{n}=4-5$ per group). $* \mathrm{P}<0.05 \mathrm{compared}$ with PBS-treated OVX mice. 
AMD3100 can improve bone loss in an OVX mice model by decreasing the number of osteoclasts differentiated from BM-derived HSPCs.

We found that administration of AMD3100 resulted in an increase in the functional levels of SDF-1 in plasma compared with $\mathrm{BM}$. This result suggests that release of HSPCs from BM to blood occurred in response to increasing levels of plasma SDF-1 in blood following administration of AMD3100. In addition, no changes in the levels of osteoblast markers were detected after treatment with AMD3100. These findings indicate that mobilization of HSPCs by AMD3100 occurs through direct blockade of CXCR4-mediated sensing of the SDF-1 chemotactic gradient in $\mathrm{BM}$ and by promoting the release of SDF-1 from BM stromal cells into the circulation, similar to previous reports $(10,19)$.

Notably, we demonstrated that the total number of CFUs in blood was increased by treatment with AMD3100 in Sham and OVX mice. We also confirmed that the LSK compartment was decreased by administration of AMD3100 in BM of OVX mice. Consistent with these results, micro-CT analyses confirmed an increase in bone thickness as a result of the inhibition of osteoclastic bone resorption. These results indicate that AMD3100 prevented bone loss in OVX mice through reduction of osteoclast number onto bone surfaces. However, although mobilization and levels of SDF-1 were increased in the plasma of Sham mice, treatment with AMD3100 did not result in significant change of BMD. This is because AMD3100 has been shown to very rapidly mobilize HSPCs into blood, without altering BM niche function, with restoration to normal steady state within hours $(9,15)$. These results suggest that AMD3100 had no effect on bone density under normal steady-state BM environment not in osteoporosis.

In summary, results of this study demonstrated that AMD3100, a novel small-molecule antagonist of CXCR4, had no influence on osteoblasts and directly targeted distribution of the interaction of CXCL12/CXCR4, resulting in rapid mobilization of HSPCs into the blood circulation and prevented bone loss induced by osteoporosis. In this study, for the first time, we link bone remodeling with the regulation of AMD3100-induced mobilization of HPSCs and propose that the delicate balance of osteoblasts and osteoclasts is also a major regulator of hematopoiesis. Therefore, we suggest the potential for use of AMD3100 as an alternative therapy for treatment of osteoporosis.

\section{MATERIALS AND METHODS}

\section{Animals and treatments}

Mouse studies were approved by the Kyungpook National University Institutional Animal Care and Use Committee (IACUC). Female C57BL/6 mice were purchased from Jackson Laboratory (Bar Harbor, ME). Mice were housed in an air-conditioned room with a $12 \mathrm{hr}$ light/dark cycle at a temperature of $22 \pm 2^{\circ} \mathrm{C}$ and humidity of $45-65 \%$ and given free access to food and tap water. Mice underwent either sham surgery or OVX at 12 weeks of age and were sacrificed at 16 weeks of age. One week after surgery, sham and OVX mice received an intraperitoneal injection with AMD3100 (Sigma-Aldrich \#A5602, St. Louis, MO, Sigma Aldrich.com) (5 mg/kg/day) or PBS for 21 days. At the end of treatment, the mice were sacrificed, and blood samples were collected by cardiac puncture for the CFU assay. Femora were removed, fixed with $4 \%$ paraformaldehyde in PBS solution ( $\mathrm{pH} \mathrm{7.4)} \mathrm{for} 16 \mathrm{hr}$, and then stored $\left(4^{\circ} \mathrm{C}\right)$ at $80 \%$ ethanol for measurement of bone density.

\section{Quantitative Real-Time PCR}

RNA samples were extracted from whole BM cells of four individual animals per group and isolated from the cultured cells using the RNeasy Mini kit (Qiagen, Hilden, Germany), and the concentration was determined using a Nanodrop ND-1000 spectrophotometer. A total of $5 \mu \mathrm{g}$ of each RNA was converted to cDNA using the sprint RT complete-oligo (dT) 18 (Clontech, MountainView, CA) according to the manufacturer's guide. The cDNA was quantified using the QuantiTect SYBR Green PCR Kit (Qiagen). For each investigated transcript, a mixture of the following reaction components was prepared to the indicated end-concentration: forward primer $(5 \mathrm{pM})$, reverse primer (5 pM), and QuantiTect SYBR Green PCR Master mix. The $10 \mu \mathrm{l}$ master-mix was added to a $0.1 \mathrm{ml}$ tube, and $5 \mu \mathrm{l}$ volume, containing 100 ng reverse transcribed total RNA, was added as polymerase chain reaction (PCR) template. The tubes were closed, centrifuged, and placed into the Corbett research RG-6000 real-time PCR machine (Corbett LifeScience, Sydney, Australia). The following primers were used: Osteocalcin (Forward 5'-GGGCAATAAGGTAGTGAACAG-3', Reverse 5'-G CAGCACAGGTCCTAAATAGT-3'), Osterix (Forward 5'-GCGT ATGGCTTCTTTGTGCCT-3', Reverse 5'-AGCTCACTATGGCT CCAGTCC-3'), Runt-related transcription factor 2 (RUNX2) (Forward 5'-ATACTGGGATGAGGAATGCG-3', Reverse 5'-CC AAGAAGGCACAGACAGAA-3'), parathyroid receptor-1 (PTHR1) (Forward: 5'-GATTCTGGTGGAGGGACTGT-3', Reverse 5'-GGA TGATCCACTTCTTGTGC-3'), Atp6v0d2 (Forward 5'-CGGAAA AGAACTCGTGAAGA-3', Reverse 5'-CTGGAAGCCCAGTAAA CAGA-3'), NFATC-1 (Forward 5'-AGGTGACACTAGGGGACA CA-3', Reverse 5'-AGTCCCTTCCAAGTTTCCAC-3'), TRAP (Forward 5'-ACTTCCCCAGCCCTTACTAC-3', Reverse 5'-TCA GCACATAGCCCACACCG-3').

\section{Enzyme-Linked Immunosorbent Assay (ELISA)}

Murine plasma was collected by cardiac puncture in tubes (a 1 $\mathrm{ml}$ syringe containing $50 \mu \mathrm{l}$ of $100 \mathrm{mM}$ EDTA), and BM was flushed with PBS. After centrifugation, plasma and BM supernatants were collected, and used for detection of SDF-1 protein by ELISA (R\&D Systems, Minneapolis, MN, USA).

\section{Hematopoietic CFU assay}

Single-cell suspensions of peripheral blood (PB) after ammonium chloride lysis were plated into $35 \mathrm{~mm}$ dishes $\left(3 \times 10^{5}\right.$ 
cells/plate) with MethoCult GF M3434 (StemCell Technologies). Hematopoietic colonies were counted and scored after incubation for $12-14$ days at $37^{\circ} \mathrm{C}, 5 \% \mathrm{CO}_{2}$, as instructed by the manufacturer.

\section{Flow cytometry}

BM cells from the femurs and tibias were collected by flushing with $20 \mathrm{ml}$ PBS passed through a 25-gauge needle. After centrifugation at $1,300 \mathrm{rpm}$ for $5 \mathrm{~min}$, the supernatant was removed and the cells were then washed by ammonium chloride lysis. Cells were incubated first using a Lineage Cell Depletion Kit magnetic labeling system with the biotinylated lineage antibody cocktail (CD5, CD45R [B220], CD11b, Gr-1 [Ly-6G/C], and Ter-119) for $10 \mathrm{~min}$ at $4^{\circ} \mathrm{C}$ and anti-biotin MicroBeads (Milt-enyi Biotec) for an additional $20 \mathrm{~min}$ at $4^{\circ} \mathrm{C}$. Positive immunoselection was performed with PE/Cy7-conjugated anti-Sca-1 (BD Pharmingen), APC-conjugated anti-mouse CD117 (c-Kit) (BD Pharmingen), SAV-PB, and a FACS Aria (BD Biosciences) using a flow cytometer.

\section{Micro-computed tomography}

For micro-computed tomography (micro-CT) in vivo imaging, we sacrificed and then scanned each group of mice at $8 \mu \mathrm{m}$ resolution using the eXplore Locus scanner (GE Healthcare). In the femora, scanning regions were confined to the distal metaphysis, extending proximally $1.7 \mathrm{~mm}$ from the proximal tip of the primary spongiosa. BMD, BMC, BVF, TMD, Tb.N., Tb.Sp., Cr.BMD and Cr.BMC were applied for performance of quantitative analysis using software provided with $2.0+\mathrm{ABA}$ Micro-view of the micro-CT system.

\section{Osteoclast differentiation}

BM cells were prepared by removal from the femurs and tibias of seven-week-old mice. The bone marrow suspension was added to plates along with macrophage colony stimulating factor (M-CSF; $30 \mathrm{ng} / \mathrm{ml}$ ). After culture for $24 \mathrm{hr}$, the non-adherent cells were collected and resuspended in $\alpha$-MEM containing $10 \%$ FBS. For the osteoclastogenesis experiments, BM-derived macrophages were plated into 6-well plates at a density of $2 \times$ $10^{6}$ cells/well in $\alpha$-MEM with $10 \%$ FBS, receptor activator for nuclear factor $\kappa B$ ligand (RANKL; $100 \mathrm{ng} / \mathrm{ml}$ ) and M-CSF (30 $\mathrm{ng} / \mathrm{ml})$ in the presence or absence of AMD3100 (25 $\mu \mathrm{g} / \mathrm{ml})$ for 3 days.

\section{Histological analysis}

Femurs were fixed in $4 \%$ paraformaldehyde for $24 \mathrm{hr}$; the tissues were then decalcified in 10\% EDTA for one week, dehydrated in ethanol, embedded in paraffin, sectioned to 4- $\mu \mathrm{m}$ thickness and stained with hematoxylin and eosin (H\&E). For TRAP staining, sections were stained with $225 \mu \mathrm{M}$ Naphthol AS-MX phosphate (Sigma-Aldrich, St. Louis, MO, USA), 0.84\% $\mathrm{N}, \mathrm{N}$-dimethylformamide (Sigma-Aldrich), and $1.33 \mathrm{mM}$ Fast Red Violet LB Salt (Sigma-Aldrich) in $50 \mathrm{mM}$ sodium acetate (pH 5.0) containing $50 \mathrm{mM}$ sodium tartrate, and incubated for
$30 \mathrm{~min}$. After incubation, sections were washed in distilled water and counterstained with $1 \%$ methyl green. We performed histomorphometric analysis using the Bioquant OSTEOII Program (BIOQUANT Image Analysis Corporation, Nashville, TN, USA).

\section{Statistical analysis}

The Student's t-test was used for comparison of two groups, whereas Tukey's HSD test and Repeated Measures Analysis of Variance test were used for multi group comparisons according to the SAS statistical package (Release 9.1; SAS Institute Inc., Cary, NC). $\mathrm{P}<0.05$ was considered significant.

\section{ACKNOWLEDGEMENTS}

This work was supported by the Basic Science Research Program (2013R1A1A2008239) and Bio \& Medical Technology Development Program (2012M3A9C6049913) through the National Research Foundation of Korea (NRF) funded by the Ministry of Education, Science and Technology, Republic of Korea. Additional support for this work was provided by Biomedical Research Institute grant, Kyungpook National University Hospital (2012).

\section{REFERENCES}

1. Usui, M., Yoshida, Y., Tsuji, K., Oikawa, K., Miyazono, K., Ishikawa, I., Yamamoto, T., Nifuji, A. and Noda, M. (2004) Tob deficiency superenhances osteoblastic activity after ovariectomy to block estrogen deficiency-induced osteoporosis. Proc. Natl. Acad. Sci. U S A. 101, 6653-6658.

2. Mansour, A., Abou-Ezzi, G., Sitnicka, E., Jacobsen, S. E., Wakkach, A. and Blin-Wakkach, C. (2012) Osteoclasts promote the formation of hematopoietic stem cell niches in the bone marrow. J. Exp. Med. 209, 537-549.

3. van den Bergh, J. P., van Geel, T. A. and Geusens, P. P. (2012) Osteoporosis, frailty and fracture: implications for case finding and therapy. Nat. Rev. Rheumatol. 8, 163-172.

4. Kim, T., Ha, H., Shim, K. S., Cho, W. K. and Ma, J. Y. (2013) The anti-osteoporotic effect of Yijung-tang in an ovariectomized rat model mediated by inhibition of osteoclast differentiation. J. Ethnopharmacol. 146, 83-89.

5. Takayanagi, H. (2007) Osteoimmunology: shared mechanisms and crosstalk between the immune and bone systems. Nat. Rev. Immunol. 7, 292-304.

6. Ishii, M., Egen, J. G., Klauschen, F., Meier-Schellersheim, M., Saeki, Y., Vacher, J., Proia, R. L. and Germain, R. N. (2009) Sphingosine-1-phosphate mobilizes osteoclast precursors and regulates bone homeostasis. Nature 458, 524-528.

7. M. Z., Lee, H., Wysoczynski, M., Wan, W., Marlicz, W., Laughlin, M. J., Kucia, M., Janowska-Wieczorek, A. and Ratajczak, J. (2010) Novel insight into stem cell mobilization-plasma sphingosine-1-phosphate is a major chemoattractant that directs the egress of hematopoietic stem progenitor cells from the bone marrow and its level in pe- 
ripheral blood increases during mobilization due to activation of complement cascade/membrane attack complex. Leukemia 24, 976-985.

8. Nervi, B., Ramirez, P., Rettig, M. P., Uy, G. L., Holt, M. S., Ritchey, J. K., Prior, J. L., Piwnica-Worms, D., Bridger, G., Ley, T. J. and DiPersio, J. F. (2009) Chemosensitization of acute myeloid leukemia (AML) following mobilization by the CXCR4 antagonist AMD3100. Blood 113, 6206-6214.

9. Rettig, M. P., Ansstas, G. and DiPersio, J. F. (2012) Mobilization of hematopoietic stem and progenitor cells using inhibitors of CXCR4 and VLA-4. Leukemia 26, 34-53.

10. Winkler, I. G., Pettit, A. R., Raggatt, L. J., Jacobsen, R. N., Forristal, C. E., Barbier, V., Nowlan, B., Cisterne, A., Bendall, L. J., Sims, N. A. and Levesque, J. P. (2012) Hematopoietic stem cell mobilizing agents G-CSF, cyclophosphamide or AMD3100 have distinct mechanisms of action on bone marrow HSC niches and bone formation. Leukemia 26, 1594-1601.

11. Christopher, M. J. and Link, D. C. (2008) Granulocyte colony-stimulating factor induces osteoblast apoptosis and inhibits osteoblast differentiation. J. Bone. Miner. Res. 23, 1765-1774.

12. Dale, D. C., Cottle, T. E., Fier, C. J., Bolyard, A. A., Bonilla, M. A., Boxer, L. A., Cham, B., Freedman, M. H., Kannourakis, G., Kinsey, S. E., Davis, R., Scarlata, D., Schwinzer, B., Zeidler, C. and Welte, K. (2003) Severe chronic neutropenia: treatment and follow-up of patients in the severe chronic neutropenia international registry. Am. J. Hematol. 72, 82-93.

13. Lee, M. Y., Fukunaga, R., Lee, T. J., Lottsfeldt, J. L. and Nagata, S. (1991) Bone modulation in sustained hematopoietic stimulation in mice. Blood 77, 2135-2141.

14. Dar, A., Schajnovitz, A., Lapid, K., Kalinkovich, A., Itkin,
T., Ludin, A., Kao, W. M., Battista, M., Tesio, M., Kollet, O., Cohen, N. N., Margalit, R., Buss, E. C., Baleux, F., Oishi, S., Fujii, N., Larochelle, A., Dunbar, C. E., Broxmeyer, H. E., Frenette, P. S. and Lapidot, T. (2011) Rapid mobilization of hematopoietic progenitors by AMD3100 and catecholamines is mediated by CXCR4-dependent SDF-1 release from bone marrow stromal cells. Leukemia 25, 1286-1296.

15. Golan, K., Vagima, Y., Ludin, A., Itkin, T., Cohen-Gur, S., Kalinkovich, A., Kollet, O., Kim, C., Schajnovitz, A., Ovadya, Y., Lapid, K., Shivtiel, S., Morris, A. J., Ratajczak, M. Z. and Lapidot, T. (2012) S1P promotes murine progenitor cell egress and mobilization via S1P1-mediated ROS signaling and SDF-1 release. Blood 119, 2478-2488.

16. Pusic, I. and DiPersio, J. F. (2010) Update on clinical experience with AMD3100, an SDF-1/CXCL12-CXCR4 inhibitor, in mobilization of hematopoietic stem and progenitor cells. Curr. Opin. Hematol. 17, 319-326.

17. De Klerck, B., Geboes, L., Hatse, S., Kelchtermans, H., Meyvis, Y., Vermeire, K., Bridger, G., Billiau, A., Schols, D. and Matthys, P. (2005) Pro-inflammatory properties of stromal cell-derived factor-1 (CXCL12) in collagen-induced arthritis. Arthritis. Res. Ther. 7, R1208-1220.

18. Kollet, O., Dar, A., Shivtiel, S., Kalinkovich, A., Lapid, K. Sztainberg, Y., Tesio, M., Samstein, R. M., Goichberg, P., Spiegel, A., Elson, A. and Lapidot, T. (2006) Osteoclasts degrade endosteal components and promote mobilization of hematopoietic progenitor cells. Nat. Med. 12, 657-664.

19. Paganessi, L. A., Walker, A. L., Tan, L. L., Holmes, I., Rich, E., Fung, H. C. and Christopherson, K. W., 2nd. (2011) Effective mobilization of hematopoietic progenitor cells in G-CSF mobilization defective CD26-/- mice through AMD3100-induced disruption of the CXCL12CXCR4 axis. Exp. Hematol. 39, 384-390. 